\title{
Casimir Interaction between a Plane and a Sphere: Correction to the Proximity-Force Approximation at Intermediate Temperatures
}

\author{
Vinicius Henning ${ }^{1}\left(\mathbb{D}\right.$, Benjamin Spreng $^{2,3} \mathbb{D}^{\mathbb{D}}$, Paulo A. Maia Neto ${ }^{1}$ (D) and Gert-Ludwig Ingold ${ }^{2, *(\mathbb{D})}$ \\ 1 Instituto de Física, Universidade Federal do Rio de Janeiro, CP 68528, Rio de Janeiro 21941-909, RJ, Brazil; \\ henningufrj@gmail.com (V.H.); pamn@if.ufrj.br (P.A.M.N.) \\ 2 Institut für Physik, Universität Augsburg, 86135 Augsburg, Germany; \\ benjamin.spreng@physik.uni-augsburg.de \\ 3 Department of Electrical and Computer Engineering, University of California, Davis, CA 95616, USA \\ * Correspondence: gert.ingold@physik.uni-augsburg.de
}

check for updates

Citation: Henning, V.; Spreng, B.; Maia Neto, P.A.; Ingold, G.-L. Casimir Interaction between a Plane and a Sphere: Correction to the

Proximity-Force Approximation at Intermediate Temperatures. Universe 2021, 7, 129. http://doi.org/10.3390/ universe7050129

Academic Editor: Galina L.

Klimchitskaya

Received: 25 March 2021

Accepted: 28 April 2021

Published: 3 May 2021

Publisher's Note: MDPI stays neutral with regard to jurisdictional claims in published maps and institutional affiliations.

Copyright: (c) 2021 by the authors. Licensee MDPI, Basel, Switzerland. This article is an open access article distributed under the terms and conditions of the Creative Commons Attribution (CC BY) license (https:// creativecommons.org/licenses/by/ $4.0 /)$

\begin{abstract}
We consider the Casimir interaction energy between a plane and a sphere of radius $R$ at finite temperature $T$ as a function of the distance of closest approach $L$. Typical experimental conditions are such that the thermal wavelength $\lambda_{T}=\hbar c / k_{\mathrm{B}} T$ satisfies the condition $L \ll \lambda_{T} \ll R$. We derive the leading correction to the proximity-force approximation valid for such intermediate temperatures by developing the scattering formula in the plane-wave basis. Our analytical result captures the joint effect of the spherical geometry and temperature and is written as a sum of temperature-dependent logarithmic terms. Surprisingly, two of the logarithmic terms arise from the Matsubara zero-frequency contribution.
\end{abstract}

Keywords: Casimir effect; scattering approach; plane-sphere geometry; thermal corrections

\section{Introduction}

The Casimir effect is a striking consequence of the zero-point energy of the quantum electromagnetic field. The geometry studied by Casimir himself was given by two planar perfectly-reflecting plates in vacuum, which experience an attractive force [1,2]. However, due to parallelism issues, most experiments are performed using either a plane-sphere [3-10] or a sphere-sphere [11-13] geometry (for reviews see [14-18]).

In contrast to the well understood plane-plane geometry, an exact theoretical formalism for the plane-sphere $[19,20]$ and the sphere-sphere [21] geometries became available only with the advent of the scattering approach [22,23]. However, experimental data for the Casimir force continued to be compared with theoretical results obtained within the proximity-force approximation (PFA) due to Derjaguin [24] as numerical implementations of the scattering formula for experimentally relevant geometrical aspect ratios were not available until very recently [25-28]. Within the PFA, the Casimir energy is obtained from Lifshitz's formula for parallel planes [29-32] by averaging over the local surface-to-surface distance [33].

Starting from the exact scattering approach for spherical surfaces, the PFA result was obtained [34] as the leading asymptotics for large sphere radius $R$ from a WKB saddlepoint contribution [35-38]. The saddle point has a direct physical interpretation in terms of specular reflection at the points of closest approach on each interacting surface [34]. Carrying out the semiclassical approximation up to the next-to-leading order, the zerotemperature leading order correction to PFA $[39,40]$ was re-derived and shown to be mostly due to corrections to the WKB approximation for the Mie scattering amplitudes [41].

Investigations of the leading-order correction to PFA became particularly relevant on account of recent experiments $[13,42-45]$ probing larger aspect ratios $L / R$ where $L$ represents the distance of closest approach. In those experiments, thermal effects have to 
be considered since the contribution from thermal photons becomes more important as the distance $L$ is increased [46-50], especially when modelling experiments with colloidal suspensions [11,12] with a near index matching at non-zero Matsubara frequencies [51]. Thus, a theoretical approach taking into account both thermal and beyond-PFA geometrical effects is required in most cases where a measurable deviation from PFA is expected.

In this paper, we derive the analytical leading-order correction to the PFA result for intermediate temperatures satisfying $L \ll \lambda_{T} \ll R$, where $\lambda_{T}=\hbar c / k_{\mathrm{B}} T$ is the thermal wavelength. Such condition holds in typical Casimir experiments as $\lambda_{T} \approx 7.6 \mu \mathrm{m}$ at $T=300 \mathrm{~K}$. We consider the plane-sphere setup within the perfectly-reflecting model for simplicity. However, our method based on a semiclassical expansion developed in the plane-wave basis $[34,41]$ can also be applied to the sphere-sphere geometry and to real materials.

The non-trivial interplay between geometrical and thermal corrections was numerically demonstrated for a scalar field model within the worldline approach [52,53]. Finitetemperature numerical implementations of the scattering approach based either on spherical multipoles $[25,26,54-58]$ or plane waves [28] provided further evidence that the thermal and curvature effects are strongly correlated. The high-temperature limit is amenable to analytical [59] and numerical $[60,61]$ calculations based on bispherical coordinates. In the case of perfect reflectors, the leading-order corrections to PFA for low temperature [62], $L \ll R \ll \lambda_{T}$, and high temperatures [60], $\lambda_{T} \ll L \ll R$, were derived analytically by considering the asymptotic limit of the scattering matrices in the multipolar spherical basis.

The derivative expansion provides yet another method to obtain the leading-order correction to PFA $[40,63]$. It relies on a re-summation of the perturbative expansion around the parallel-planes geometry [64]. The derivative expansion is implemented by approximating the perturbative kernel by its power series up to second order in the momentum variable $k$. For a scalar field satisfying Neumann boundary conditions, the finite-temperature kernel is not analytical at $k=0$ in the case of three spatial dimensions, and then the derivative expansion breaks down [65]. This is also the case for the electromagnetic Casimir effect in three dimensions when considering perfectly-reflecting or plasma mirrors [66]. The singular behavior of the perturbative kernel indicates that the correction to PFA is of a nonlocal nature at finite temperatures.

Such non-analytical and nonlocal behavior translates into a correction to PFA containing powers of $\log (L / R)$, as first discussed in connection with the high-temperature regime [67]. We show that Bimonte's $\log ^{2}(L / R)$ leading correction arising from the Matsubara zero-frequency contribution [60], which is usually associated to the high-temperature regime, should also be kept when $L \ll \lambda_{T} \ll R$. By developing the scattering formula in the plane-wave basis, we re-derive Bimonte's result as well as the next-to-leading order correction proportional to $\log (L / R)$. The latter turns out to be also required for an accurate description of experimentally relevant aspect ratios. For the contribution of the non-zero Matsubara frequencies, we derive a correction proportional to $\log ^{2}\left(L / \lambda_{T}\right)$ by employing the Euler-Maclaurin sum formula.

The paper is organized as follows. Section 2 presents in a first part the basic tools and notations required to expand the scattering formula in the plane-wave basis. A second part discusses the asymptotic expansion in powers of the inverse sphere radius and introduces general expressions for the leading-order correction to PFA. Section 3 is devoted to an explicit evaluation of the leading-order correction for individual Matsubara frequencies. A particular focus will be put on the special case of the zero Matsubara frequency. The results from this section will be used in Section 4 to derive the leading-order correction to PFA valid for intermediate temperatures. In the analysis, we will distinguish between the contributions arising from the geometric optical WKB approximation and from its diffraction correction. Concluding remarks are presented in Section 5. Appendix A presents the derivation of the WKB expansion for the Mie scattering amplitudes at nonzero imaginary frequencies, which is a key ingredient in our approach. A review of the next-to-leading term for the 
saddle-point approximation for the one-dimensional case together with the results for the multidimensional generalization is given in Appendix $B$.

\section{Asymptotic Expansion of the Casimir Free Energy in the Plane-Wave Basis}

\subsection{Casimir Free Energy for Plane-Sphere Geometry}

We consider a spherical surface of radius $R$ close to a plate as illustrated in Figure 1 and assume both surfaces to be perfectly reflecting. The plate lies in the $x y$-plane and the $z$-axis perpendicular to it goes through the sphere center. The closest distance between plate and sphere is denoted by $L$.

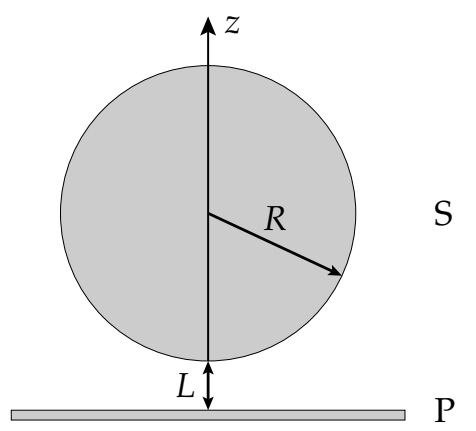

Figure 1. Sphere (S) of radius $R$ and plate $(\mathrm{P})$ separated by a distance $L$.

Within the scattering approach [22], the Casimir free energy is written as a sum over the Matsubara frequencies $\xi_{n}=2 \pi n k_{\mathrm{B}} T / \hbar$

$$
\mathcal{F}=\sum_{n=0}^{\infty}{ }^{\prime} \mathcal{F}_{n}
$$

with

$$
\mathcal{F}_{n}=k_{\mathrm{B}} T \operatorname{tr} \log \left[1-\mathcal{M}\left(\xi_{n}\right)\right]
$$

and where the prime indicates that the term $n=0$ is multiplied by one-half. The operator $\mathcal{M}\left(\xi_{n}\right)$ describes the round trip of an electromagnetic wave in the empty gap between the two interacting surfaces. For the geometry shown in Figure 1, the round-trip operator is decomposed as

$$
\mathcal{M}\left(\xi_{n}\right)=\mathcal{T}_{\mathrm{PS}} \mathcal{R}_{\mathrm{S}} \mathcal{T}_{\mathrm{SP}} \mathcal{R}_{\mathrm{P}}
$$

$\mathcal{R}_{\mathrm{S}}$ and $\mathcal{R}_{\mathrm{P}}$ are the reflection operators for sphere and plate taken with respect to reference points at the sphere center and at the intersection between the $z$-axis and the plate surface, respectively. $\mathcal{T}_{\text {PS }}$ describes the translation from the first to the second reference point over a distance $L+R$ along the $z$-axis, while $\mathcal{T}_{\text {SP }}$ accounts for the translation in the opposite direction.

We expand the logarithm in (2) in powers of the round-trip operator $\mathcal{M}\left(\xi_{n}\right)$ :

$$
\mathcal{F}_{n}=-k_{\mathrm{B}} T \sum_{r=1}^{\infty} \frac{1}{r} \operatorname{tr} \mathcal{M}\left(\xi_{n}\right)^{r} .
$$

The summation variable $r$ in (4) represents the number of round trips between the two interacting surfaces. Thus, the Casimir free energy collects all the contributions from one to infinitely many round trips within the empty cavity bounded by the reflecting surfaces.

We evaluate the trace in (4) in the plane-wave basis $\{|\mathbf{k}, \phi, p\rangle\}$ as defined by the angular spectral representation [68]. Here, $\mathbf{k}$ denotes the projection of the wave vector onto the $x y$-plane, $\phi= \pm 1$ defines the sense of propagation along the $z$-axis in upwards or downwards direction, respectively, and the polarization $p$ is either transverse electric (TE) or transverse magnetic (TM). The projected wave vector $\mathbf{k}$ and the Matsubara frequency $\xi_{n}$ jointly define the Wick-rotated axial component of the three-dimensional wave vector 


$$
\kappa=\sqrt{\frac{\xi_{n}^{2}}{c^{2}}+\mathbf{k}^{2}} .
$$

The translation operators $\mathcal{T}_{\mathrm{PS}}$ and $\mathcal{T}_{\mathrm{SP}}$ are diagonal in the plane-wave basis with eigenvalues $e^{-\kappa(R+L)}$. The action of the reflection operator at the planar surface

$$
\mathcal{R}_{\mathrm{P}}|\mathbf{k},-, p\rangle=r_{p}|\mathbf{k},+, p\rangle,
$$

conserves the projected wave vector $\mathbf{k}$ as well as the polarization $p$. Here, $r_{p}$ are the standard Fresnel coefficients for specular reflection which, for the case of perfect reflectors, are given by $r_{\mathrm{TM}}=1$ and $r_{\mathrm{TE}}=-1$.

In contrast, $\mathbf{k}$ and $p$ are not conserved during a reflection at the spherical surface. The contribution corresponding to $r$ round-trips in (4) apart from the trace thus implies an integration over $r-1$ intermediate wave vectors $\mathbf{k}_{1}, \ldots, \mathbf{k}_{r-1}$ and a summation over intermediate polarizations $p_{1}, \ldots, p_{r-1}$ taking values TE or TM

$$
\operatorname{tr} \mathcal{M}\left(\xi_{n}\right)^{r}=\sum_{p_{0}, \ldots, p_{r-1}} \int \prod_{j=0}^{r-1} \frac{\mathrm{d} \mathbf{k}_{j}}{(2 \pi)^{2}} e^{-2 \kappa_{j}(L+R)} r_{p_{j}}\left\langle\mathbf{k}_{j+1},-, p_{j+1}\left|\mathcal{R}_{S}\right| \mathbf{k}_{j},+, p_{j}\right\rangle .
$$

We use a cyclic index convention such that $j=r$ is equivalent to $j=0$. The matrix elements of the reflection operator $\mathcal{R}_{\mathrm{S}}$ appearing in (7) can be written in terms of the standard Mie scattering amplitudes together with coefficients describing the change between the Fresnel and the scattering polarization basis [34,41]. For the evaluation of the leading-order (LO) PFA result and its LO correction for the perfectly reflectors case, the relevant matrix elements effectively reduce to [41]

$$
\begin{aligned}
\left\langle\mathbf{k}_{j},-, \mathrm{TM}\left|\mathcal{R}_{\mathrm{S}}\right| \mathbf{k}_{i},+, \mathrm{TM}\right\rangle & =\frac{2 \pi c}{\xi \kappa_{j}} S_{2} \\
\left\langle\mathbf{k}_{j},-, \mathrm{TE}\left|\mathcal{R}_{\mathrm{S}}\right| \mathbf{k}_{i},+, \mathrm{TE}\right\rangle & =\frac{2 \pi c}{\xi \kappa_{j}} S_{1}
\end{aligned}
$$

while the matrix elements involving the coupling between different polarizations do not contribute, i.e., $\left\langle\mathbf{k}_{j}, \mathrm{TM}\left|\mathcal{R}_{S}\right| \mathbf{k}_{i}, \mathrm{TE}\right\rangle=\left\langle\mathbf{k}_{j}, \mathrm{TE}\left|\mathcal{R}_{S}\right| \mathbf{k}_{i}, \mathrm{TM}\right\rangle=0$. The Mie scattering amplitudes $S_{1}$ and $S_{2}$ in (8) are given by (A1) of Appendix A. They are functions of the imaginary size parameter $\xi R / c$ and the scattering angle $\Theta$ defined through

$$
\cos (\Theta)=-\frac{c^{2}}{\xi^{2}}\left(\kappa_{i} \kappa_{j}+\mathbf{k}_{i} \cdot \mathbf{k}_{j}\right) .
$$

As discussed in Appendix A, the Mie scattering amplitudes can be expanded for large $R$ as [35-38]

$$
S_{p}=S_{p}^{\mathrm{WKB}}\left(1+\frac{1}{R} s_{p}+O\left(R^{-2}\right)\right) .
$$

with the leading-order contribution given by the WKB expression

$$
S_{p}^{\mathrm{WKB}}=(-1)^{p} \frac{\xi R}{2 c} \exp \left[\frac{2 \xi R}{c} \sin \left(\frac{\Theta}{2}\right)\right]
$$

and

$$
\begin{aligned}
& s_{1}=\frac{c}{2 \xi} \frac{\cos (\Theta)}{\sin ^{3}(\Theta / 2)}=-\frac{\sqrt{2}\left(\kappa_{i} \kappa_{j}+\mathbf{k}_{i} \cdot \mathbf{k}_{j}\right)}{\left(\xi^{2} / c^{2}+\kappa_{i} \kappa_{j}+\mathbf{k}_{i} \cdot \mathbf{k}_{j}\right)^{3 / 2}} \\
& s_{2}=-\frac{c}{2 \xi} \frac{1}{\sin ^{3}(\Theta / 2)}=-\frac{\sqrt{2} \xi^{2} / c^{2}}{\left(\xi^{2} / c^{2}+\kappa_{i} \kappa_{j}+\mathbf{k}_{i} \cdot \mathbf{k}_{j}\right)^{3 / 2}} .
\end{aligned}
$$


describing the leading-order corrections.

\subsection{Asymptotic Expansion}

Evaluating the trace (7) within the lowest-order saddle-point approximation (LO-SPA) together with the WKB expression (11) for the Mie scattering amplitudes, one obtains by means of (1) and (4) the Casimir free energy within the proximity-force approximation [34]. This result constitutes the leading term in an asymptotic expansion for large sphere radius $R$ and can be entirely understood in terms of geometrical optics.

Our aim is to go beyond the proximity-force approximation and to determine the corrections which are smaller by a factor $1 / R$. Two corrections need to be taken into account. Firstly, in the evaluation of the trace (7) one needs to go one order beyond the LO-SPA. We refer to this correction as next-to-leading order saddle-point approximation (NTLO-SPA). Since this correction is not as widely known as the LO-SPA, we give some details in Appendix B. In the evaluation of the NTLO-SPA, the Mie scattering amplitudes are still to be taken within the WKB approximation and we are thus still within the realm of geometrical optics. A second contribution to the correction to the proximity-force approximation arises from the leading correction to the WKB Mie scattering amplitudes as specified by (10) together with (12) and (13). This contribution takes diffraction into account. For this second contribution, it is sufficient to evaluate the integrals in (7) within LO-SPA.

Inserting (10) in (7), allows us to express the trace over the $r$-th round trip in the form

$$
\operatorname{tr} \mathcal{M}\left(\xi_{n}\right)^{r} \simeq\left(\frac{R}{4 \pi}\right)^{r} \int \mathrm{d} \mathbf{k}_{0} \ldots \mathrm{d} \mathbf{k}_{r-1} g\left(\mathbf{k}_{0}, \ldots, \mathbf{k}_{r-1}\right) e^{-R f\left(\mathbf{k}_{0}, \ldots, \mathbf{k}_{r-1}\right)}
$$

with

$$
g\left(\mathbf{k}_{0}, \ldots, \mathbf{k}_{r-1}\right)=\sum_{p=1,2} \prod_{j=0}^{r-1} \frac{e^{-2 \kappa_{j} L}}{\kappa_{j}}\left(1+\frac{1}{R} s_{p}\right)
$$

and

$$
f\left(\mathbf{k}_{0}, \ldots, \mathbf{k}_{r-1}\right)=\sum_{j=0}^{r-1}\left(\kappa_{j}+\kappa_{j+1}-\left[2\left(\xi_{n}^{2} / c^{2}+\kappa_{j} \kappa_{j+1}+\mathbf{k}_{j} \cdot \mathbf{k}_{j+1}\right)\right]^{1 / 2}\right) .
$$

Note that $s_{p}$ in (15) depends on the indices $j$ and $j+1$ through the respective wave vectors.

The $2 r$-dimensional integral in (14) is suitable for a saddle-point approximation where $R$ plays the role of the large parameter. It is straightforward to show that there exists a continuous family of saddle points

$$
\mathbf{k}_{0}=\cdots=\mathbf{k}_{r-1} \equiv \mathbf{k}_{\mathrm{sp}}
$$

parameterized by $\mathbf{k}_{\mathrm{sp}}$. While the saddle-point approximation can be applied in the directions orthogonal to the saddle-point manifold, in the end we will be left with an integral over the saddle-point manifold which needs to be evaluated exactly.

As a consequence of the existence of a continuous family of saddle points, the Hessian matrix of (16) is singular with two vanishing eigenvalues in view of the two-dimensional character of $\mathbf{k}_{\mathrm{sp}}$. In order to cope with the vanishing eigenvalues, it is convenient to transform the Hessian matrix into its eigenbasis as described in Ref. [41]. For completeness, we review in the following the basic steps.

On the saddle-point manifold (17), the Hessian matrix can be brought into blockdiagonal form

$$
\mathbf{H}=\left(\begin{array}{cc}
\mathrm{H}_{x x} & 0 \\
0 & \mathrm{H}_{y y}
\end{array}\right)
$$

with the matrix elements

$$
\left(\mathrm{H}_{x x}\right)_{i j}=\left.\frac{\partial^{2} f}{\partial k_{i, x} \partial k_{j, x}}\right|_{\mathrm{sp}}
$$


and a corresponding expression for $\mathrm{H}_{y y}$.

The blocks of the Hessian matrix can be expressed as $\mathrm{H}_{x x}=\mathrm{H}_{y y}=\left(1 / 2 \kappa_{\mathrm{sp}}\right) \Gamma_{r}$ in terms of the $r \times r$ circulant matrix

$$
\Gamma_{r}=\left(\begin{array}{ccccc}
2 & -1 & & & -1 \\
-1 & 2 & -1 & & \\
& -1 & \ddots & \ddots & \\
& & \ddots & \ddots & -1 \\
-1 & & & -1 & 2
\end{array}\right)
$$

for $r \geq 3$ and where the matrix elements not shown are zero. In the case of two round trips

$$
\Gamma_{2}=\left(\begin{array}{cc}
2 & -2 \\
-2 & 2
\end{array}\right)
$$

(note that the corresponding expression in [41] is missing a factor of 2) while for $r=1$ $f \equiv 0$.

It is now convenient to introduce transformed variables $v$ through

$$
k_{j, x}=\sum_{l=0}^{r-1} \mathrm{~W}_{j l} v_{l, x}
$$

with the Fourier matrix

$$
\mathrm{W}_{j l}=\frac{1}{\sqrt{r}} \exp \left(\frac{2 \pi i}{r} j l\right)
$$

and correspondingly for the $y$-direction.

After the transformation, the two blocks of the Hessian matrix are of counter-diagonal form

$$
\left(\mathrm{W}^{T} \mathrm{H}_{x x} \mathrm{~W}\right)_{j l}=\lambda_{j} \delta_{j, r-l}
$$

with the eigenvalues

$$
\lambda_{j}=\frac{2}{\kappa_{\mathrm{sp}}} \sin ^{2}\left(\frac{\pi j}{r}\right)
$$

and $j=0,1, \ldots, r-1$. As expected, one eigenvalue $(j=0)$ vanishes for each block and the variables $v_{0, x}$ and $v_{0, y}$ parametrize the two-dimensional saddle-point manifold.

Applying the saddle-point approximation (A14) with (A16) and (A18), (14) can now be expressed as [41]

$$
\operatorname{tr} \mathcal{M}\left(\xi_{n}\right)^{r}=\frac{R}{2 r} \int_{\xi_{n} / c}^{\infty} \mathrm{d} \kappa_{\mathrm{sp}} \kappa_{\mathrm{sp}}^{r}\left[F_{0}+\frac{1}{R} F_{1}+o\left(R^{-1}\right)\right],
$$

where we have transformed the variables $v_{0, x}$ and $v_{0, y}$ back to the wave vector at the saddle point. Note that in contrast to the big-O symbol used earlier, the little-o symbol is used to denote terms which change asymptotically faster than indicated by the argument [69]. Here, the last term collects all terms decaying faster than $R^{-1}$ for large radii without specifying the precise dependency on $R$.

The first and second terms in the integrand of (26) correspond to the LO-SPA and NTLO-SPA, respectively, and are given by

$$
F_{0}=\left.g\right|_{\mathrm{sp}}
$$

and

$$
F_{1}=\left.g\right|_{\mathrm{sp}}\left(\sum_{i j k} \frac{f_{i j k} f_{\bar{i} \bar{j} \bar{k}}}{12 \lambda_{i} \lambda_{j} \lambda_{k}}-\sum_{i j} \frac{f_{i \bar{i} j \bar{j}}}{8 \lambda_{i} \lambda_{j}}\right)+\sum_{i} \frac{g_{i \bar{i}}}{2 \lambda_{i}}
$$


where we have introduced the shorthand notation $\bar{i}=r-i$. The summation runs over the indices from 1 to $r-1$ and implies also a summation over the corresponding components $x$ and $y$. The indices at the functions $f$ and $g$ denote derivatives with respect to the corresponding components of the variables $v$ evaluated at the saddle point. Note that in comparison with (A18) the second and the fourth term are missing which were shown in Ref. [41] not to contribute to (28).

\section{Leading-Order Correction for Individual Matsubara Frequencies}

For an asymptotic expansion in powers of the inverse sphere radius, the radius $R$ has to be compared with the other length scales of the problem. While the radius can be chosen larger than $c / \xi_{n}$ for all $n>0$, the zero Matsubara frequency $\xi_{0}$ may require special care. Interestingly, it turns out that the leading order terms for the matrix elements (8) and as a consequence for the trace (7) hold for all Matsubara frequencies. The expressions for the zero Matsubara frequency can thus be obtained by taking the zero-frequency limit of the results for positive Matsubara frequencies. Therefore, the PFA result holds for arbitrary temperatures including the high-temperature limit determined by the contribution of the zero Matsubara frequency [34].

The situation is different when the next-to-leading order term is considered. In contrast to the contributions due to positive Matsubara frequencies, the zero-frequency contribution cannot be obtained from the known diffraction correction [35-38] to the WKB Mie scattering amplitudes (11). Proceeding on that basis would yield an infrared divergence in the corresponding integral (26) over $k_{\mathrm{sp}}$.

We will start by discussing the case of positive Matsubara frequencies in Section 3.1 where we make use of results obtained earlier in Ref. [41]. In Section 3.2, we will then derive the asymptotic expansion of the zero-frequency contribution to obtain both the NTLO and the next-to-next-to-leading order (NNTLO) terms. The latter turns out to be non-negligible for experimentally relevant aspect ratios and then should be kept alongside the former, which was first derived in Ref. [60] by the multipolar approach. In Section 3.2, we focus on the TE zero-frequency contribution, as the TM correction can be more easily derived from an exact analytical representation obtained either by using bispherical coordinates [59] or by developing the plane-wave basis representation (7) [70].

\subsection{Positive Matsubara Frequencies}

We first turn to the discussion of Matsubara frequencies $\xi_{n}$ with $n>0$ and consider the two contributions to the integrand in (26). The leading-order term (27) is obtained by evaluating (15) at the saddle point and can be decomposed into contributions from the two polarizations as

$$
F_{0}=g_{\mathrm{TE}}+g_{\mathrm{TM}}
$$

with

$$
\begin{aligned}
g_{\mathrm{TE}} & =\frac{e^{-2 r \kappa_{\mathrm{sp}} L}}{\kappa_{\mathrm{sp}}^{r}}\left(1+\frac{r\left(\xi_{n}^{2}-2 c^{2} \kappa_{\mathrm{sp}}^{2}\right)}{2 c^{2} \kappa_{\mathrm{sp}}^{3} R}\right) \\
g_{\mathrm{TM}} & =\frac{e^{-2 r \kappa_{\mathrm{sp}} L}}{\kappa_{\mathrm{sp}}^{r}}\left(1-\frac{r \xi_{n}^{2}}{2 c^{2} \kappa_{\mathrm{sp}}^{3} R}\right) .
\end{aligned}
$$

The evaluation of the next-order term (28) is more involved. We refer the reader to Appendix A in [41] for details. There, it was found that

$$
F_{1}=-\frac{\left(r^{2}-1\right)\left(r L \kappa_{\mathrm{sp}}\left(c^{2} \kappa_{\mathrm{sp}}^{2}+\xi_{n}^{2}\right)+\xi_{n}^{2}\right)}{6 r c^{2} \kappa_{\mathrm{sp}}^{3}} \frac{e^{-2 r \kappa_{\mathrm{sp}} L}}{\kappa_{\mathrm{sp}}^{r}} .
$$

The leading term in the $1 / R$ expansion corresponding to the PFA result is determined entirely by local scattering channels describing specular reflection at the point of closest 
approach on the spherical surface. It can thus be completely understood in terms of geometrical optics. In contrast, the NTLO term consists of two contributions

$$
\left[\operatorname{tr} \mathcal{M}\left(\xi_{n}\right)^{r}\right]_{\mathrm{NTLO}}=\sum_{p=\mathrm{TE}, \mathrm{TM}}\left(\left[\operatorname{tr} \mathcal{M}\left(\xi_{n}\right)^{r}\right]_{\mathrm{d}}^{p}+\left[\operatorname{tr} \mathcal{M}\left(\xi_{n}\right)^{r}\right]_{\mathrm{go}}^{p}\right)
$$

of different physical origin. The first term carrying the subscript " $\mathrm{d}$ " captures the effect of diffraction as it arises from the LO correction to the WKB approximation for the Mie scattering amplitudes taken at the LO-SPA. The second term with subscript "go" is still calculated within the LO geometric optical WKB approximation and contains the NTLOSPA. Physically, it amounts to displacing the point where specular reflection takes place from the point of closest approach between the two surfaces. Note that taking the diffraction contribution (which is already a NTLO term) into account within the NTLO-SPA would lead to a higher order contribution which can be neglected here.

In correspondence with the zero-temperature results derived in Ref. [41], the different NTLO contributions obtained from the expansion of (7) for an individual Matsubara frequency are given by

$$
\begin{aligned}
{\left[\operatorname{tr} \mathcal{M}\left(\xi_{n}\right)^{r}\right]_{\mathrm{d}}^{\mathrm{TE}} } & =\frac{1}{8}\left[\left(u^{2}-4\right) E_{1}(u)-(u-1) e^{-u}\right] \\
{\left[\operatorname{tr} \mathcal{M}\left(\xi_{n}\right)^{r}\right]_{\mathrm{d}}^{\mathrm{TM}} } & =-\frac{1}{8}\left[u^{2} E_{1}(u)-(u-1) e^{-u}\right] \\
{\left[\operatorname{tr} \mathcal{M}\left(\xi_{n}\right)^{r}\right]_{\mathrm{go}}^{p} } & =-\frac{\left(r^{2}-1\right) e^{-u}}{12 r^{2}}, p=\mathrm{TE}, \mathrm{TM} .
\end{aligned}
$$

Here, $E_{1}$ denotes the exponential integral function [71] and $u=2 L r \xi_{n} / c$. The two polarizations provide identical contributions to the geometrical optics term.

After inserting (33)-(35) into the contribution (4) of an individual Matsubara frequency to the Casimir free energy, we sum over multiple round-trips to find the NTLO contribution for any non-zero Matsubara frequency

$$
\left[\mathcal{F}_{n}\right]_{\mathrm{NTLO}}=\left[\mathcal{F}_{n}\right]_{\mathrm{d}}^{\mathrm{TE}}+\left[\mathcal{F}_{n}\right]_{\mathrm{d}}^{\mathrm{TM}}+\left[\mathcal{F}_{n}\right]_{\text {go }} \quad(n \neq 0)
$$

consisting of the contributions from diffraction

$$
\begin{aligned}
{\left[\mathcal{F}_{n}\right]_{\mathrm{d}}^{\mathrm{TE}} } & =-\left[\mathcal{F}_{n}\right]_{\mathrm{d}}^{\mathrm{TM}}-\frac{\hbar c}{2 \lambda_{T}} \int_{1}^{\infty} \mathrm{d} t \frac{\log \left(1-e^{-4 \pi \tau n t}\right)}{t} \\
{\left[\mathcal{F}_{n}\right]_{\mathrm{d}}^{\mathrm{TM}} } & =\frac{\hbar c}{8 \lambda_{T}}\left[(4 \pi \tau n)^{2} \int_{1}^{\infty} \mathrm{d} t \frac{e^{-4 \pi \tau n t}}{t\left(1-e^{-4 \pi \tau n t}\right)^{2}}-4 \pi \tau n \frac{e^{-4 \pi \tau n}}{1-e^{-4 \pi \tau n}}-\log \left(1-e^{-4 \pi \tau n}\right)\right]
\end{aligned}
$$

and from geometrical optics

$$
\left[\mathcal{F}_{n}\right]_{\text {go }}^{p}=-\frac{\hbar c}{12 \lambda_{T}}\left[\operatorname{Li}_{3}\left(e^{-4 \pi \tau n}\right)+\log \left(1-e^{-4 \pi \tau n}\right)\right], p=\text { TE, TM, }
$$

where $\mathrm{Li}_{3}$ denotes the trilogarithm [71]. These results are valid for arbitrary values of the ratio $\tau=L / \lambda_{T}$ as long as $R \gg L, \lambda_{T}$. In Section 4 , when considering the case of intermediate temperatures $R \gg \lambda_{T} \gg L$, we will expand (37)-(39) for $\tau \ll 1$.

\subsection{Zero Matsubara Frequency}

We now turn to the zero-frequency contribution $\mathcal{F}_{0}$ to the Casimir free energy and determine the corrections to the PFA result. At vanishing frequency, the reflection matrix elements of the sphere are diagonal with respect to polarization [34]. For the TM contribution, the plane-wave approach allows for the derivation of an exact analytic expression in the more general case of two spheres of arbitrary radii [70]. The previously known result 
for the plane-sphere geometry [59] is recovered as a particular case. The leading order PFA correction is then found to be proportional to $\log (L / R)$

$$
\mathcal{F}_{0}^{\mathrm{TM}} \simeq-\frac{k_{\mathrm{B}} T}{4}\left[\frac{\zeta(3)}{x}-\frac{1}{6} \log (x)+o(\log (x))\right],
$$

where we have introduced the dimensionless quantity $x=L / R$ and $\zeta(3) \approx 1.202$ denotes a particular value of the Riemann zeta function [71]. In the remaining part of this section, we focus on the asymptotic expansion of the TE contribution to $\mathcal{F}_{0}$ when the sphere radius $R$ becomes large compared to the surface-to-surface distance $L$.

The low-frequency limit of the reflection operator at the sphere has been derived in [34]. With Equations (8), (A9) and (B6) of Ref. [34], the matrix elements for TE polarization read

$$
\left\langle\mathbf{k}_{j+1},-, \mathrm{TE}\left|\mathcal{R}_{\mathrm{S}}\right| \mathbf{k}_{j},+, \mathrm{TE}\right\rangle=\frac{2 \pi R}{k_{j+1}} \sum_{\ell=1}^{\infty} \frac{\ell}{\ell+1} \frac{y_{j+1, j}^{2 \ell}}{(2 \ell) !}
$$

with

$$
y_{j+1, j}=R \sqrt{2\left(\mathbf{k}_{j+1} \cdot \mathbf{k}_{j}+k_{j+1} k_{j}\right)} .
$$

For large spheres, for which all $y_{j+1, j} \gg 1$, the asymptotics of the reflection matrix elements (41) can be obtained by replacing the sum over $\ell$ by an integral and using Stirling's approximation for the factorial. The asymptotics of the integral over $\ell$ can then be found by the leading-order saddle-point approximation with a saddle point at $\ell_{\mathrm{sp}}=y_{j+1, j} / 2$. For the asymptotics of the reflection matrix elements (41), we then find

$$
\left\langle\mathbf{k}_{j+1},-, \mathrm{TE}\left|\mathcal{R}_{\mathrm{S}}\right| \mathbf{k}_{j},+, \mathrm{TE}\right\rangle=\frac{\pi R}{k_{j+1}} \frac{y_{j+1, j}}{y_{j+1, j}+2} e^{y_{j+1, j}}\left(1+O\left(\frac{1}{R^{2}}\right)\right) .
$$

Formally, the zero-frequency limit of (10) could be reproduced by expanding the second factor. However, we need to keep the full expression to avoid a divergence in the integrals (45) and (52) below. When applying the saddle-point approximation to (14), the function (15) thus has to be replaced by

$$
g\left(\mathbf{k}_{0}, \ldots, \mathbf{k}_{r-1}\right)=\prod_{j=0}^{r-1} \frac{e^{-2 k_{j} L}}{k_{j}} \frac{y_{j+1, j}}{y_{j+1, j}+2}
$$

while in (16) it is sufficent to set $\xi_{n}=0$.

We now evaluate the contributions to (26) due to LO-SPA and NTLO-SPA separately. For the LO-SPA of the trace over $r$ round trips, we then find

$$
\left[\operatorname{tr} \mathcal{M}(0)^{r}\right]_{\mathrm{LO}-\mathrm{SPA}}^{\mathrm{TE}} \simeq \frac{1}{2 r x} \int_{0}^{\infty} \mathrm{d} t\left(\frac{t}{t+x}\right)^{r} e^{-2 r t},
$$

where we have substituted $t=k_{\mathrm{sp}} L$. Since the sphere radius is much larger than the distance between plane and sphere, we can approximate the integrand in (45) for $x \ll 1$ and write

$$
\begin{aligned}
{\left[\operatorname{tr} \mathcal{M}(0)^{r}\right]_{\mathrm{LO}-\mathrm{SPA}}^{\mathrm{TE}} } & \simeq \frac{1}{2 r x} \int_{0}^{\infty} \mathrm{d} t \exp [-r(2 t+x / t)] \\
& =\frac{1}{r \sqrt{2 x}} K_{1}(2 r \sqrt{2 x})
\end{aligned}
$$

in terms of the modified Bessel function of the second kind $K_{1}$ [71]. The terms neglected here contribute to higher order in the asymptotic expansion.

In view of (4) we need to evaluate the sum over the number $r$ of round trips of (46) weighted with an additional factor $1 / r$. The presence of the Bessel function leads us to 
employ a method based on the Mellin transformation [72]. The round-trip sum can then be expressed as an integral

$$
\sum_{r=1}^{\infty} \frac{K_{1}(2 r \sqrt{2 x})}{r^{2}}=\frac{1}{8 \pi \mathrm{i}} \int_{c-\mathrm{i} \infty}^{c+\mathrm{i} \infty} \mathrm{d} s \Gamma\left(\frac{s-1}{2}\right) \Gamma\left(\frac{s-3}{2}\right) \zeta(s)(2 x)^{-s / 2}
$$

where $\Gamma(z)$ is the Gamma function [71] and the integration contour has to be chosen such that $c>3$. The integrand contains a single pole at $s=3$, a triple pole at $s=1$ and double poles at $s=-2 n+1$ with $n=1,2, \ldots$ Keeping only the pole at $s=3$ is equivalent to PFA and the logarithmic corrections which we are interested in arise from the pole at $s=1$. Evaluating the corresponding residues and neglecting terms of order one and higher, we find the asymptotic expansion of the free energy due to the LO-SPA as

$$
\left[\mathcal{F}_{0}^{\mathrm{TE}}\right]_{\mathrm{LO}-\mathrm{SPA}} \simeq-\frac{k_{\mathrm{B}} T}{4}\left[\frac{\zeta(3)}{x}-\frac{1}{2} \log ^{2}(x)+(1-\log (2)) \log (x)+O(1)\right] .
$$

For the NTLO-SPA, we need to evaluate (28). It turns out that we can partly use the results obtained for finite frequencies in Ref. [41]. Thus, the expressions for the derivatives of the function $f$ are obtained from (A13) and (A14) of Ref. [41] by taking $\xi \rightarrow 0$. One can show that the contributions to $F_{1}$ arising from the derivatives of $f$ cancel out.

In the remaining term in (28), the function $g$ defined in (44) is differentiated with respect to $v_{i}$ and $v_{r-i}$. This term can be decomposed into two contributions,

$$
F_{1}=\frac{D_{3,1}+D_{3,2}}{2}
$$

$D_{3,1}$ and $D_{3,2}$ correspond to double derivatives of the two factors $\exp \left(-2 k_{j} L\right) / k_{j}$ and $y_{j+1, j} /\left(y_{j+1, j}+2\right)$, respectively. The contribution where a single derivative is taken of each of those factors vanishes.

The term $D_{3,1}$ can be obtained from (A15) of Ref. [41] by taking the zero-frequency limit. We then find

$$
D_{3,1}=-\left.\frac{\left(r^{2}-1\right) L}{3} g\right|_{\mathrm{sp}} .
$$

In order to determine the term $D_{3,2}$, we follow the procedure described in Appendix A of Ref. [41] and find

$$
D_{3,2}=-\left.\frac{(r-1)\left(3+(r+1) k_{\mathrm{sp}} R\right)}{6 k_{\mathrm{sp}}\left(1+k_{\mathrm{sp}} R\right)^{2}} g\right|_{\mathrm{sp}} .
$$

The NTLO-SPA of the trace over $r$ round trips can then be expressed as

$$
\left[\operatorname{tr} \mathcal{M}(0)^{r}\right]_{\mathrm{NTLO}-\mathrm{SPA}}^{\mathrm{TE}}=-\frac{r-1}{12 r} \int_{0}^{\infty} \mathrm{d} t\left[r+1+x \frac{3 x+(r+1) t}{2 t(x+t)^{2}}\right]\left(\frac{t}{t+x}\right)^{r} e^{-2 r t},
$$

where we again used the substitution $t=k_{\mathrm{sp}} L$. For $x \ll 1$, we can write

$$
\begin{aligned}
{\left[\operatorname{tr} \mathcal{M}(0)^{r}\right]_{\mathrm{NTLO}-\mathrm{SPA}}^{\mathrm{TE}} } & \simeq-\frac{r^{2}-1}{12 r} \int_{0}^{\infty} \mathrm{d} t\left[1+\frac{x}{2 t^{2}}\right] e^{-r(2 t+x / t)} \\
& =-\frac{r^{2}-1}{6 r} \sqrt{2 x} K_{1}(2 r \sqrt{2 x})
\end{aligned}
$$

Note that the second term in the square bracket above needs to be kept, as it is asymptotically of the same order as the first one. Performing the sum over round trips using the method of Ref. [72], we find the NTLO saddle-point contribution to the Casimir free energy for TE polarization as

$$
\left[\mathcal{F}_{0}^{\mathrm{TE}}\right]_{\mathrm{NTLO}-\mathrm{SPA}} \simeq-\frac{k_{\mathrm{B}} T}{24} \log (x) .
$$


The total TE contribution to the free energy then becomes

$$
\begin{aligned}
\mathcal{F}_{0}^{\mathrm{TE}} & \simeq\left[\mathcal{F}_{0}^{\mathrm{TE}}\right]_{\mathrm{LO}-\mathrm{SPA}}+\left[\mathcal{F}_{0}^{\mathrm{TE}}\right]_{\mathrm{NTLO}-\mathrm{SPA}} \\
& =-\frac{k_{\mathrm{B}} T}{4}\left[\frac{\zeta(3)}{x}-\frac{1}{2} \log ^{2}(x)+\left(\frac{7}{6}-\log (2)\right) \log (x)+o(\log (x))\right] .
\end{aligned}
$$

To verify that the asymptotic expression for the zero-frequency contribution to the Casimir free energy due to TE polarization given by (55) is correct, we compare with the corresponding numerically exact result. In Figure 2, the difference between the asymptotics and the numerical exact result is shown as a function of $x=L / R$. As this difference is decreasing with decreasing values of $x$ and thus subleading compared to $\log (x)$, our numerical comparison shows that the asymptotic expansion (55) is indeed correct.

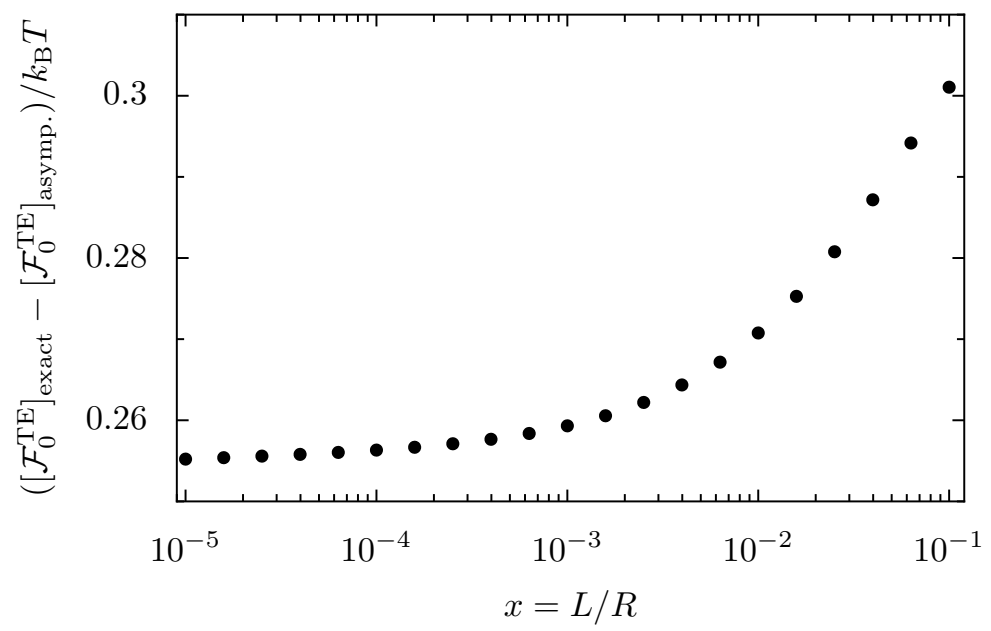

Figure 2. Numerical analysis of the correction to formula (55). The dots show the difference between the numerically exact values of the zero-frequency contribution to the Casimir free energy for TE polarization and the corresponding values according to formula (55). This difference is shown as a function of $x=L / R$.

The complete asymptotic expansion for the zero-frequency contribution is obtained by adding the contributions of the TM polarization (40) and the TE polarization (55) and will explicitly be given and used in the next section in (60).

\section{Leading-Order Correction to PFA at Intermediate Temperatures}

The contribution of thermal fluctuations to the leading-order correction to the PFA result for the Casimir free energy is derived as the difference between the Matsubara sum of $\left[\mathcal{F}_{n}\right]_{\text {NTLO }}$ and the corresponding integral representing the zero-temperature limit. Such a difference is usually evaluated with the help of the Abel-Plana [73] or the Poisson summation [50] formula. Since the zero-frequency contribution was treated separately in the previous section, we found it more convenient to make use of the Euler-Maclaurin formula in the form

$$
\sum_{n=1}^{\infty} \mathcal{F}_{n}=\int_{1}^{\infty} \mathrm{d} n \mathcal{F}_{n}+\frac{\mathcal{F}_{1}+\mathcal{F}_{\infty}}{2}+\sum_{m=1}^{\infty} \frac{B_{2 m}\left[\mathcal{F}_{\infty}^{(2 m-1)}-\mathcal{F}_{1}^{(2 m-1)}\right]}{(2 m) !},
$$

where $\mathcal{F}_{n}^{(2 m-1)}$ denotes the $(2 m-1)$-th derivative of $\mathcal{F}_{n}$ with respect to $n$ taken as a continuous variable and $B_{2 m}$ are the Bernoulli numbers [71].

We apply the Euler-Maclaurin formula (56) to the sum of the NTLO Matsubara contributions $\left[\mathcal{F}_{n}\right]_{\text {NTLO }}$ given by Equations (36)-(39). These terms decay for large frequencies so that $\left[\mathcal{F}_{\infty}\right]_{\text {NTLO }}$ and all its derivatives vanish. Only the first Matsubara frequency contributes 
to the second and third terms in (56). For $\tau=L / \lambda_{T} \ll 1$, the leading contribution to $\left[\mathcal{F}_{1}\right]_{\mathrm{NTLO}}$ arises from the TE diffraction term (37)

$$
\left[\mathcal{F}_{1}\right]_{\mathrm{d}}^{\mathrm{TE}} \simeq \frac{\hbar c \tau}{4 L} \log ^{2}(\tau)
$$

Relative to the zero-temperature NTLO result, the TE diffraction contribution is $O\left(\tau \log ^{2}(\tau)\right)$, whereas both the TM diffractive contribution (38) and the geometric optical contributions (39) are $O(\tau \log \tau)$. In addition, the derivatives of $\left[\mathcal{F}_{1}\right]_{\text {NTLO }}$ appearing in the Euler-Maclaurin formula (56) are also $O(\tau \log \tau)$ and hence can be neglected.

In order to connect the integral appearing on the right-hand side of (56) with the zero-temperature result, we need to account for the difference in the lower bound. Thus, we obtain the NTLO terms from (56) as

$$
\sum_{n=1}^{\infty}\left[\mathcal{F}_{n}\right]_{\mathrm{NTLO}} \simeq[\mathcal{F}(T=0)]_{\mathrm{NTLO}}-\int_{0}^{1} \mathrm{~d} n\left[\mathcal{F}_{n}\right]_{\mathrm{NTLO}}+\frac{\left[\mathcal{F}_{1}\right]_{\mathrm{d}}^{\mathrm{TE}}}{2}
$$

where $[\mathcal{F}(T=0)]_{\text {NTLO }}$ denotes the NTLO contribution to the free energy in the zerotemperature limit. The integral subtracted on the right-hand side of (58) is of the same order as $\left[\mathcal{F}_{1}\right]_{\mathrm{d}}^{\mathrm{TE}}$ and its leading-order contribution also arises from the TE diffraction term (37). We find

$$
\sum_{n=1}^{\infty}\left[\mathcal{F}_{n}\right]_{\mathrm{NTLO}} \simeq[\mathcal{F}(T=0)]_{\mathrm{NTLO}}-\frac{\hbar c \tau}{8 L} \log ^{2}(\tau) .
$$

Finally, it is still necessary to add the Matsubara zero-frequency contribution $\left[\mathcal{F}_{0}\right]_{\text {NTLO }}$ to (59) in order to obtain the full NTLO Casimir free energy from (1). Naively, one could expect that $\left[\mathcal{F}_{0}\right]_{\text {NTLO }}$ would not contribute in the limit $\tau \ll 1$. However, this term is relevant for intermediate temperatures $L / R \ll \tau \ll 1$ as far as the correction to PFA is concerned. In Section 3.2, we have found that the zero-frequency contribution to the Casimir free energy with (40) and (55) reads, up to NNTLO,

$$
\mathcal{F}_{0} \simeq-\frac{\hbar c \tau}{4 L}\left[\frac{2 \zeta(3)}{x}-\frac{1}{2} \log ^{2}(x)+(1-\log (2)) \log (x)+O(1)\right],
$$

where $x=L / R$ was introduced at the beginning of Section 3.2. The NTLO and NNTLO zero-frequency contributions correspond to the second and third terms on the right-hand side of (60). They are both asymptotically larger than the thermal correction arising from nonzero frequencies given by (59) when $x \ll \tau \ll 1$. In practice, however, all those contributions are comparable in the case of experimentally relevant values of $\tau$ and $x$, as illustrated by the numerical example discussed below.

As we want to focus on the interplay between geometrical and thermal effects, we first define the total thermal correction to the Casimir free energy

$$
\delta \mathcal{F}(T)=\mathcal{F}(T)-\mathcal{F}(T=0) .
$$

and introduce the deviation of the thermal correction from the PFA result relative to the zero-temperature PFA free energy

$$
\Delta=\frac{\delta \mathcal{F}(T)-\delta \mathcal{F}_{\mathrm{PFA}}(T)}{\mathcal{F}_{\mathrm{PFA}}(T=0)} .
$$

After taking (59) and (60) into account, we find for intermediate temperatures $x \ll \tau \ll 1$

$$
\Delta \simeq \frac{45}{\pi^{3}} x \tau\left[-\log ^{2}(x)+2[1-\log (2)] \log (x)+2 \log ^{2}(\tau)+O(\log (\tau))\right],
$$

where the leading neglected terms arise from non-zero Matsubara frequencies. 
In Figure 3, we show the correction $\Delta$ as a function of temperature. The geometrical aspect ratio is chosen as $x=L / R=10^{-3}$, a typical order-of-magnitude in most Casimir experiments [26]. In the upper panel (Figure 3a), the full Matsubara sum is considered. The dots represent the exact correction as calculated by the numerical method presented in Ref. [28], whereas the line corresponds to the analytical approximation (63). In contrast, in the lower panel (Figure 3b), the contribution of the zero Matsubara frequency has been disregarded. Note that the sign of $\Delta$ in the two panels differs.

According to the results displayed in Figure 3b, the sum over nonzero frequencies is well described by the analytical formula derived from (59) in the range of intermediate temperatures $x \ll \tau \ll 1$. The resulting correction is positive, thus reducing the total correction to PFA. In contrast, the total thermal contribution to the PFA correction, with the zero-frequency contribution included, is negative in the entire range shown in the figure. Thus, the strength of the interaction is further reduced with respect to the PFA prediction due to thermal effects. The zero-temperature result underestimates the total correction to PFA by a factor of about two at $\tau \approx 3 \times 10^{-2}$, indicating the strong interplay between thermal and geometrical effects $[52,53]$.
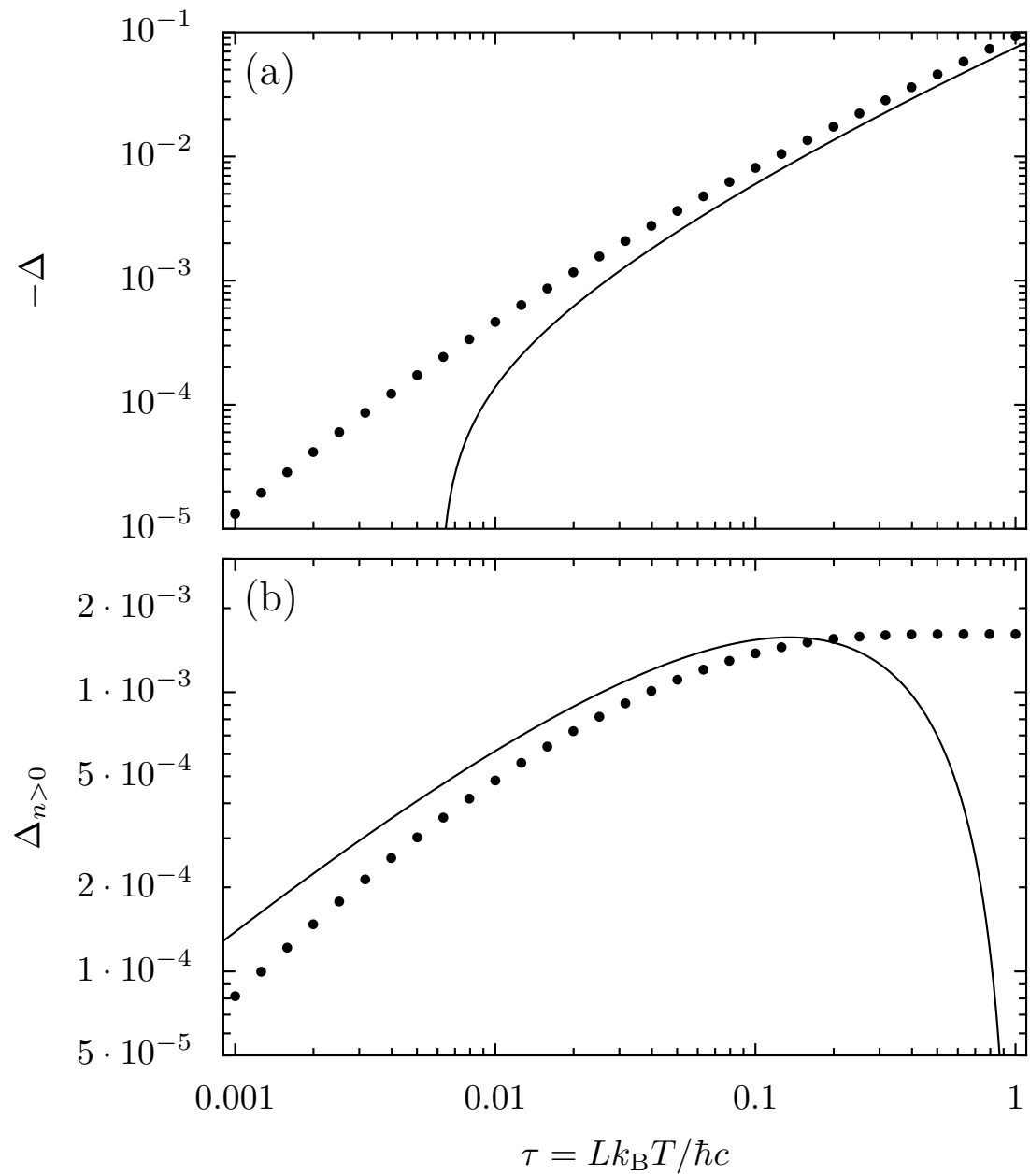

Figure 3. Relative thermal correction (62) of the Casimir free energy as a function of temperature for a geometrical aspect ratio of $x=L / R=10^{-3}$. (a) The dots represent the numerically exact full Matsubara sum and the line corresponds to the analytical asymptotic expansion (63). Note that the correction $\Delta$ is negative. (b) Contribution $\Delta_{n>0}$ of the positive Matsubara frequencies $n>0$. The dots again represent the numerically exact result while the line corresponds to the analytical asymptotic expansion derived from (59). For $n>0$, the correction to the PFA result is positive.

The zero-frequency contribution plays a significant role in such interplay, as the total PFA correction and the thermal contribution from nonzero frequencies have opposite signs 
in the entire range shown in Figure 3a. We find good agreement between the data and our analytical formula (63) for $x \ll \tau$. Since the zero-frequency contribution becomes increasingly dominant as the temperature rises above $\tau \sim 0.1$, formula (63) also provides a good description even beyond the range of intermediate temperatures.

The results obtained in this section allow for the derivation of the NTLO Casimir entropy for intermediate temperatures. By adding formula (60) to (59) and neglecting sub-leading contributions when taking the derivative with respect to temperature, we find

$$
S_{\mathrm{NTLO}} \simeq \frac{k_{\mathrm{B}}}{16}\left[2 \log ^{2}(\tau)-\log ^{2}\left(\frac{L}{R}\right)+2(1-\log (2)) \log \left(\frac{L}{R}\right)\right] .
$$

The first term on the right-hand side results from the contribution of nonzero frequencies. The zero-frequency contribution, represented by the second and third terms, corresponds to a temperature-independent, negative contribution reminiscent of the negative Casimir entropies found for aspect ratios $L / R \sim 1$ or larger [55,58].

\section{Conclusions}

We have analyzed the leading-order correction to PFA in the plane-sphere geometry for intermediate temperatures satisfying the condition $x=L / R \ll \tau=L k_{\mathrm{B}} T / \hbar c \ll 1$, which holds in most Casimir force experiments. Whereas the Matsubara zero frequency is unimportant for extremely low temperatures satisfying $\tau \ll x \ll 1$, it provides a sizeable contribution to the correction in the case of intermediate temperatures. When considering its asymptotic limit for $R \gg L$, we should keep not only Bimonte's NTLO term [60], proportional to $x \tau \log ^{2}(x)$, but also the NNTLO term proportional to $x \tau \log (x)$ in order to have an accurate formula for experimentally relevant aspect ratios. We have also derived an additional logarithmic term of the form $x \tau \log ^{2}(\tau)$ by considering the contribution of nonzero frequencies. As an effect of the logarithmic terms, the zero-temperature result grossly underestimates the correction to PFA even at the rather low temperatures $\tau \sim 10^{-2}$ corresponding to typical experiments. Altogether our findings demonstrate the strong interplay between thermal and beyond-PFA geometrical corrections.

Author Contributions: V.H. and B.S. developed the theoretical formalism and B.S. carried out the numerical calculations. P.A.M.N. and G.-L.I. coordinated the work. All authors discussed the results and co-wrote the manuscript. All authors have read and agreed to the published version of the manuscript.

Funding: Deutscher Akademischer Austauschdienst (DAAD); Conselho Nacional de Desenvolvimento Científico e Tecnológico (CNPq), Coordenação de Aperfeiçoamento de Pessoal de Nível Superior (CAPES); Instituto Nacional de Ciência e Tecnologia Fluidos Complexos (INCT-FCx); Fundação Carlos Chagas Filho de Amparo à Pesquisa do Estado do Rio de Janeiro (FAPERJ); Fundação de Amparo à Pesquisa do Estado de São Paulo (FAPESP).

Data Availability Statement: The data represented in Figures 2 and 3 are freely available from Zenodo at https:/ / doi.org/10.5281/zenodo.4631940 [74].

Acknowledgments: The authors would like to thank Michael Hartmann and Tanja Schoger for stimulating discussions. This work has been supported by CAPES and DAAD through the PROBRAL collaboration program.

Conflicts of Interest: The authors declare no conflict of interest.

\section{Abbreviations}

The following abbreviations are used in this manuscript:

PFA proximity-force approximation

LO leading order

LO-SPA leading order saddle-point approximation

NTLO next-to-leading order 
NTLO-SPA next-to-leading order saddle-point approximation

NNTLO next-to-next-to-leading order

TE transverse electric

TM transverse magnetic

WKB Wentzel-Kramers-Brillouin

\section{Appendix A. Mie Scattering Amplitudes in the WKB Approximation}

In this Appendix, we present the derivation of the asymptotic WKB expansion for the Mie scattering amplitudes [75]

$$
\begin{aligned}
& S_{1}(\Theta)=\sum_{\ell=1}^{\infty} \frac{2 \ell+1}{\ell(\ell+1)}\left[a_{\ell} \pi_{\ell}(\cos (\Theta))+b_{\ell} \tau_{\ell}(\cos (\Theta))\right] \\
& S_{2}(\Theta)=\sum_{\ell=1}^{\infty} \frac{2 \ell+1}{\ell(\ell+1)}\left[a_{\ell} \tau_{\ell}(\cos (\Theta))+b_{\ell} \pi_{\ell}(\cos (\Theta))\right],
\end{aligned}
$$

for polarizations perpendicular and parallel to the scattering plane, respectively. The angular functions $\pi_{\ell}$ and $\tau_{\ell}$ are defined by [75]

$$
\begin{aligned}
\pi_{\ell}(z) & =P_{\ell}^{\prime}(z) \\
\tau_{\ell}(z) & =-\left(1-z^{2}\right) P_{\ell}^{\prime \prime}(z)+z P_{\ell}^{\prime}(z)
\end{aligned}
$$

in terms of the Legendre polynomials $P_{\ell}$, with the prime denoting a derivative with respect to the argument $z$.

The Mie coefficients

$$
\begin{aligned}
& a_{\ell}(i \tilde{\xi})=\frac{\pi}{2}(-1)^{\ell+1} \frac{\ell I_{\ell+1 / 2}(\tilde{\xi})-\tilde{\xi} I_{\ell-1 / 2}(\tilde{\xi})}{\ell K_{\ell+1 / 2}(\tilde{\xi})+\tilde{\xi} K_{\ell-1 / 2}(\tilde{\xi})} \\
& b_{\ell}(i \tilde{\xi})=\frac{\pi}{2}(-1)^{\ell+1} \frac{I_{\ell+1 / 2}(\tilde{\xi})}{K_{\ell+1 / 2}(\tilde{\xi})} .
\end{aligned}
$$

are the electric and magnetic multipole scattering amplitudes, respectively, for perfect reflectors at imaginary frequencies. Here, $I_{\ell-1 / 2}(\tilde{\xi})$ and $K_{\ell-1 / 2}(\tilde{\xi})$ denote the modified Bessel functions of the first and second kinds [71], respectively. We consider the limit of large radius so that the size parameter $\tilde{\xi} \equiv \xi R / c \gg 1$. A separate, specific derivation for the zero-frequency case is presented in detail in Appendix B of Ref. [34].

The scattering angle (9) is of the form $\Theta=\pi+i v$, with $v$ a non-negative real number such that $\cos \Theta=-\cosh v$. According to the localization principle [37], the multipole order scales as $\ell \sim \tilde{\xi} \gg 1$. As a consequence, we approximate the sum over multipoles in (A1) by an integral over $\lambda \equiv(\ell+1 / 2) / \tilde{\xi}$. Using the uniform asymptotic expansion for large orders [76] of the Legendre polynomials in (A2) and of the modified Bessel functions in (A3), we find (see [77] for details)

$$
\begin{aligned}
S_{p}(\Theta) & \approx \int_{0}^{\infty} d \lambda g_{\text {Mie }}^{(p)}(\lambda) \exp \left[-\tilde{\xi} f_{\text {Mie }}(\lambda)\right], p=1,2 \\
f_{\text {Mie }}(\lambda) & =2 \lambda \sinh ^{-1}(\lambda)-2 \sqrt{1+\lambda^{2}}-v \lambda \\
g_{\text {Mie }}^{(p)}(\lambda) & =(-1)^{p} \tilde{\xi}^{3 / 2}\left(\frac{\lambda}{2 \pi \sinh v}\right)^{1 / 2}\left(1+h_{\text {Mie }}^{(p)}(\lambda) \frac{1}{\tilde{\xi}}\right),
\end{aligned}
$$

with the auxiliary function

$$
h_{\mathrm{Mie}}^{(p)}(\lambda)=\left(\frac{1}{\sinh v}-\frac{7}{8} \operatorname{coth} v\right) \frac{1}{\lambda}+\frac{1}{4}\left[\frac{1}{\sqrt{1+\lambda^{2}}}+\left(2(-1)^{p}+\frac{1}{3}\right) \frac{\lambda^{2}}{\left(1+\lambda^{2}\right)^{3 / 2}}\right]
$$

containing the NTLO terms in the expansion of the summand in (A1) for $\tilde{\xi} \gg 1$. 
We evaluate (A4) within the saddle-point approximation. The saddle point $\lambda_{s}$ is determined by the condition $f_{\text {Mie }}^{\prime}\left(\lambda_{s}\right)=0$. From (A5), we find

$$
\lambda_{s}=\sinh (v / 2)
$$

A similar derivation holds for real frequencies [35-38], in which case the saddle point has a direct physical interpretation in terms of the impact parameter leading to reflection, according to geometrical optics, along the direction determined by the scattering angle [78]. The second derivative at the saddle point

$$
f_{\text {Mie }}^{\prime \prime}\left(\lambda_{s}\right)=\frac{2}{\cosh (v / 2)} .
$$

is a positive real number, thus allowing for a direct application of the results for the LO-SPA and NTLO-SPA outlined in Appendix B. We obtain Equations (10)-(13) of Section 2.1 by expressing the final results for the scattering amplitudes in terms of the complex scattering angle $\Theta$.

\section{Appendix B. Next-to-Leading-Order Correction in the Saddle-Point Approximation}

In the main part of this paper, we need to asymptotically evaluate an integral of the form

$$
I=\int \mathrm{d}^{d} \mathbf{x} g(\mathbf{x}) \exp (-R f(\mathbf{x}))
$$

for large values $R$ where $\mathbf{x}=\left(x_{1}, \ldots, x_{d}\right)$ is a $d$-dimensional vector. To keep the discussion simple, we start with the one-dimensional case and merely state the result for the multidimensional case at the end. Furthermore, we will assume the existence of only a single saddle point (sp), i.e., a point where the first derivative $f^{\prime}(x)$ vanishes, and this point should lie well inside the range of integration. This will be the case in our application.

Using Laplace's method, one obtains the well-known leading order of the saddle-point approximation (LO-SPA) of the integral (A10) as

$$
I_{\mathrm{LO}-\mathrm{SPA}}=\left(\frac{2 \pi}{R f_{\mathrm{sp}}^{\prime \prime}}\right)^{1 / 2} g_{\mathrm{sp}} \exp \left(-R f_{\mathrm{sp}}\right) .
$$

We assume here that the second derivative $f_{\mathrm{sp}}^{\prime \prime}$ at the saddle point is positive. $f_{\mathrm{sp}}$ and $g_{\mathrm{sp}}$ denote the value of the functions $f(x)$ and $g(x)$, respectively, at the saddle point.

For our purposes, we also need the next-to-leading-order term of the saddle-point approximation (NTLO-SPA) which relative to the LO-SPA carries an additional factor $1 / R$ and which we will derive now. For a nonvanishing second derivative $f_{\text {sp }}^{\prime \prime}$ only a region of width $R^{-1 / 2}$ around the saddle point contributes to the integral (A10). We therefore extend the Taylor expansion in the exponent up to fourth order and expand the exponential containing the third and fourth order terms into a Taylor series. Keeping only terms contributing to the LO-SPA and the NTLO-SPA after integration, we can approximate the exponential by

$$
\begin{aligned}
\exp (-R f(x)) \approx & \exp \left(-R f_{\mathrm{sp}}\right) \exp \left[-\frac{R}{2} f_{\mathrm{sp}}^{\prime \prime} x^{2}\right] \\
& \times\left(1-\frac{R}{6} f_{\mathrm{sp}}^{\prime \prime \prime} x^{3}-\frac{R}{24} f_{\mathrm{sp}}^{\prime \prime \prime \prime} x^{4}+\frac{R^{2}}{72} f_{\mathrm{sp}}^{\prime \prime \prime 2} x^{6}\right) .
\end{aligned}
$$

Here, we have assumed for simplicity that the saddle point is located at $x=0$. In addition, we need to expand the function $g(x)$ up to second order

$$
g(x) \approx g_{\mathrm{sp}}+g_{\mathrm{sp}}^{\prime} x+\frac{1}{2} g_{\mathrm{sp}}^{\prime \prime} x^{2} .
$$


Inserting (A12) and (A13) into (A10) for $d=1$, the integration can be carried out and we obtain

$$
I=I_{\mathrm{LO}-\mathrm{SPA}}+\frac{1}{R} I_{\mathrm{NTLO}-\mathrm{SPA}}+O\left(R^{-2}\right)
$$

with

$$
I_{\text {NTLO-SPA }}=I_{\text {LO-SPA }}\left(\frac{1}{2} \frac{g_{\text {sp }}^{\prime \prime}}{g_{\mathrm{sp}} f_{\mathrm{sp}}^{\prime \prime}}-\frac{1}{2} \frac{g_{\mathrm{sp}}^{\prime} f_{\mathrm{sp}}^{\prime \prime \prime}}{g_{\mathrm{sp}} f_{\mathrm{sp}}^{\prime \prime 2}}-\frac{1}{8} \frac{f_{\mathrm{sp}}^{\prime \prime \prime \prime}}{f_{\mathrm{sp}}^{\prime \prime 2}}+\frac{5}{24} \frac{f_{\mathrm{sp}}^{\prime \prime \prime 2}}{f_{\mathrm{sp}}^{\prime \prime 3}}\right) .
$$

In the multi-dimensional case, the generalization of the result (A11) for the leading order is well-known to read

$$
I_{\mathrm{LO}-\mathrm{SPA}}=\left(\frac{2 \pi}{R}\right)^{d / 2} \frac{e^{-R f_{\mathrm{sp}}}}{\sqrt{\operatorname{det} \mathrm{H}}} g_{\mathrm{sp}}
$$

with the Hessian matrix

$$
\mathrm{H} \equiv\left(\left.\frac{\partial^{2} f}{\partial x_{i} \partial x_{j}}\right|_{\mathrm{sp}}\right)_{i, j=1, \ldots, d}
$$

which is assumed to be non-singular. Proceeding along the lines explained for the onedimensional case, the next-to-leading term in the saddle-point approximation becomes

$$
\begin{aligned}
I_{\text {NTLO-SPA }}=I_{\text {LO-SPA }}\left[\frac{1}{2} \frac{g_{i j} \mathrm{H}^{i j}}{g_{\text {sp }}}-\frac{1}{2} \frac{f_{i j k} g_{l} \mathrm{H}^{i j} \mathrm{H}^{k l}}{g_{\text {sp }}}-\frac{1}{8} f_{i j k l} \mathrm{H}^{i j} \mathrm{H}^{k l}\right. \\
\left.+\frac{1}{24} f_{i j k} f_{l m n}\left(3 \mathrm{H}^{i j} \mathrm{H}^{k l} \mathrm{H}^{m n}+2 \mathrm{H}^{i l} \mathrm{H}^{j m} \mathrm{H}^{k n}\right)\right]
\end{aligned}
$$

where the subscript "sp" denotes the evaluation of the function at $\mathbf{x}=\mathbf{x}_{\mathrm{sp}}$. A derivative with respect to the $i$-th component of $\mathbf{x}$ with subsequent evaluation at the saddle-point is represented by a lower index $i: f_{i} \equiv \partial f /\left.\partial x_{i}\right|_{\mathbf{x}=\mathbf{x}_{\mathbf{s p}}}$ and equivalently for $g$. Likewise, higher-order derivatives are denoted by multiple lower indices. Two upper indices denote the matrix elements of the inverse matrix, $\mathrm{H}^{i j} \equiv\left(\mathrm{H}^{-1}\right)_{i j}$, and the Einstein summation convention is implied, i.e., indices occuring both as sub- and superscript within a term are summed over with values running from 1 to $d$. The relation between the result (A18) and the one-dimensional result (A15) is rather straightforward except for the last two terms in (A18). They account for different index pairings and collapse into a single term in the one-dimensional case, i.e., the last term in (A15).

\section{References}

1. Casimir, H.B.G. On the attraction between two perfectly conducting plates. Proc. Kon. Ned. Akad. Wet. 1948, 51, 793-795.

2. Bordag, M.; Klimchitskaya, G.L.; Mohideen, U.; Mostepanenko, V.M. Advances in the Casimir Effect; Oxford University Press: Oxford, UK, 2009. [CrossRef]

3. Sushkov, A.O.; Kim, W.J.; Dalvit, D.A.R.; Lamoreaux, S.K. Observation of the thermal Casimir force. Nat. Phys. 2011, 7, 230-233. [CrossRef]

4. Torricelli, G.; Pirozhenko, I.; Thornton, S.; Lambrecht, A.; Binns, C. Casimir force between a metal and a semimetal. EPL 2011, 93, 51001. [CrossRef]

5. Chang, C.C.; Banishev, A.A.; Castillo-Garza, R.; Klimchitskaya, G.L.; Mostepanenko, V.M.; Mohideen, U. Gradient of the Casimir force between Au surfaces of a sphere and a plate measured using an atomic force microscope in a frequency-shift technique. Phys. Rev. B 2012, 85, 165443. [CrossRef]

6. Garcia-Sanchez, D.; Fong, K.Y.; Bhaskaran, H.; Lamoreaux, S.; Tang, H.X. Casimir Force and In Situ Surface Potential Measurements on Nanomembranes. Phys. Rev. Lett. 2012, 109, 027202. [CrossRef]

7. Banishev, A.A.; Klimchitskaya, G.L.; Mostepanenko, V.M.; Mohideen, U. Demonstration of the Casimir Force between Ferromagnetic Surfaces of a Ni-Coated Sphere and a Ni-Coated Plate. Phys. Rev. Lett. 2013, 110, 137401. [CrossRef]

8. Sedighi, M.; Svetovoy, V.B.; Palasantzas, G. Casimir force measurements from silicon carbide surfaces. Phys. Rev. B 2016, 93, 085434. [CrossRef]

9. Bimonte, G.; López, D.; Decca, R.S. Isoelectronic determination of the thermal Casimir force. Phys. Rev. B 2016, 93, 184434. [CrossRef] 
10. Cunuder, A.L.; Petrosyan, A.; Palasantzas, G.; Svetovoy, V.; Ciliberto, S. Measurement of the Casimir force in a gas and in a liquid. Phys. Rev. B 2018, 98. [CrossRef]

11. Elzbieciak-Wodka, M.; Popescu, M.N.; Ruiz-Cabello, F.J.M.; Trefalt, G.; Maroni, P.; Borkovec, M. Measurements of dispersion forces between colloidal latex particles with the atomic force microscope and comparison with Lifshitz theory. J. Chem. Phys. 2014, 140, 104906. [CrossRef]

12. Ether, D.S., Jr.; Pires, L.B.; Umrath, S.; Martinez, D.; Ayala, Y.; Pontes, B.; de S Araújo, G.R.; Frases, S.; Ingold, G.-L.; Rosa, F.S.S.; et al. Probing the Casimir force with optical tweezers. EPL 2015, 112, 44001. [CrossRef]

13. Garrett, J.L.; Somers, D.A.T.; Munday, J.N. Measurement of the Casimir Force between Two Spheres. Phys. Rev. Lett. 2018, 120, 040401. [CrossRef]

14. Klimchitskaya, G.L.; Mohideen, U.; Mostepanenko, V.M. The Casimir force between real materials: Experiment and theory. Rev. Mod. Phys. 2009, 81, 1827-1885. [CrossRef]

15. Decca, R.; Aksyuk, V.; López, D. Casimir Force in Micro and Nano Electro Mechanical Systems. Lect. Notes Phys. 2011, 834, 287-309. [CrossRef]

16. Lamoreaux, S.K. Progress in Experimental Measurements of the Surface-Surface Casimir Force: Electrostatic Calibrations and Limitations to Accuracy. Lect. Notes Phys. 2011, 834, 219-248. [CrossRef]

17. Klimchitskaya, G.L.; Mostepanenko, V.M. Recent measurements of the Casimir force: Comparison between experiment and theory. Mod. Phys. Lett. A 2020, 35, 2040007. [CrossRef]

18. Gong, T.; Corrado, M.R.; Mahbub, A.R.; Shelden, C.; Munday, J.N. Recent progress in engineering the Casimir effect-Applications to nanophotonics, nanomechanics, and chemistry. Nanophotonics 2021, 10, 523. [CrossRef]

19. Emig, T. Fluctuation-induced quantum interactions between compact objects and a plane mirror. J. Stat. Mech. 2008, 2008, P04007. [CrossRef]

20. Maia Neto, P.A.; Lambrecht, A.; Reynaud, S. Casimir energy between a plane and a sphere in electromagnetic vacuum. Phys. Rev. A 2008, 78, 012115. [CrossRef]

21. Emig, T.; Graham, N.; Jaffe, R.L.; Kardar, M. Casimir Forces between Arbitrary Compact Objects. Phys. Rev. Lett. 2007, 99, 170403. [CrossRef]

22. Lambrecht, A.; Maia Neto, P.A.; Reynaud, S. The Casimir effect within scattering theory. New J. Phys. 2006, 8, 243. [CrossRef]

23. Rahi, S.J.; Emig, T.; Graham, N.; Jaffe, R.L.; Kardar, M. Scattering theory approach to electrodynamic Casimir forces. Phys. Rev. D 2009, 80, 085021. [CrossRef]

24. Derjaguin, B. Untersuchungen über die Reibung und Adhäsion, IV-Theorie des Anhaftens kleiner Teilchen. Kolloid-Z. 1934, 69, 155-164. [CrossRef]

25. Hartmann, M.; Ingold, G.-L.; Maia Neto, P.A. Plasma versus Drude Modeling of the Casimir Force: Beyond the Proximity Force Approximation. Phys. Rev. Lett. 2017, 119, 043901. [CrossRef]

26. Hartmann, M.; Ingold, G.-L.; Maia Neto, P.A. Advancing numerics for the Casimir effect to experimentally relevant aspect ratios. Phys. Scr. 2018, 93, 114003. [CrossRef]

27. Hartmann, M.; Ingold, G.-L. CaPS: Casimir Effect in the Plane-Sphere Geometry. J. Open Source Softw. 2020, 5, 2011. [CrossRef]

28. Spreng, B.; Maia Neto, P.A.; Ingold, G.-L. Plane-wave approach to the exact van der Waals interaction between colloid particles. J. Chem. Phys. 2020, 153, 024115. [CrossRef] [PubMed]

29. Lifshitz, E.M. The Theory of Molecular Attractive Forces between Solids. Sov. Phys. JETP 1956, 2, $73-83$.

30. Kats, E.I. Influence of nonlocality effects on van der Waals interaction. Sov. Phys. JETP 1977, 46, 109-113.

31. Jaekel, M.T.; Reynaud, S. Casimir force between partially transmitting mirrors. J. Phys. 1991, 1, 1395-1409. [CrossRef]

32. Genet, C.; Lambrecht, A.; Reynaud, S. Casimir force and the quantum theory of lossy optical cavities. Phys. Rev. A 2003, 67, 043811. [CrossRef]

33. Parsegian, V.A. Van der Waals Forces: A Handbook for Biologists, Chemists, Engineers, and Physicists; Cambridge University Press: Cambridge, UK, 2006. [CrossRef]

34. Spreng, B.; Hartmann, M.; Henning, V.; Maia Neto, P.A.; Ingold, G.-L. Proximity force approximation and specular reflection: Application of the WKB limit of Mie scattering to the Casimir effect. Phys. Rev. A 2018, 97, 062504. [CrossRef]

35. Nussenzveig, H.M. High-Frequency Scattering by a Transparent Sphere. I. Direct Reflection and Transmission. J. Math. Phys. 1969, 10, 82-124. [CrossRef]

36. Khare, V. Short-Wavelength Scattering of Electromagnetic Waves by a Homogeneous Sphere. Ph.D. Thesis, University of Rochester, Rochester, NY, USA, 1975.

37. Nussenzveig, H.M. Diffraction Effects in Semiclassical Scattering; Cambridge University Press: Cambridge, UK, 1992. [CrossRef]

38. Grandy, W.T., Jr. Scattering of Waves from Large Spheres; Cambridge University Press: Cambridge, UK, 2005. [CrossRef]

39. Teo, L.P.; Bordag, M.; Nikolaev, V. Corrections beyond the proximity force approximation. Phys. Rev. D 2011, 84, 125037. [CrossRef]

40. Bimonte, G.; Emig, T.; Jaffe, R.L.; Kardar, M. Casimir forces beyond the proximity approximation. EPL 2012, 97, 50001. [CrossRef]

41. Henning, V.; Spreng, B.; Hartmann, M.; Ingold, G.-L.; Maia Neto, P.A. The role of diffraction in the Casimir effect beyond the proximity force approximation. J. Opt. Soc. Am. B 2019, 36, C77-C87. [CrossRef]

42. Krause, D.E.; Decca, R.S.; López, D.; Fischbach, E. Experimental Investigation of the Casimir Force beyond the Proximity-Force Approximation. Phys. Rev. Lett. 2007, 98, 050403. [CrossRef] 
43. Liu, M.; Xu, J.; Klimchitskaya, G.L.; Mostepanenko, V.M.; Mohideen, U. Examining the Casimir puzzle with an upgraded AFM-based technique and advanced surface cleaning. Phys. Rev. B 2019, 100. [CrossRef]

44. Liu, M.; Xu, J.; Klimchitskaya, G.L.; Mostepanenko, V.M.; Mohideen, U. Precision measurements of the gradient of the Casimir force between ultraclean metallic surfaces at larger separations. Phys. Rev. A 2019, 100, 052511. [CrossRef]

45. Bimonte, G.; Spreng, B.; Maia Neto, P.A.; Ingold, G.-L.; Klimchitskaya, G.L.; Mostepanenko, V.M.; Decca, R.S. Measurement of the Casimir Force between 0.2 and $8 \mu \mathrm{m}$ : Experimental Procedures and Comparison with Theory. Universe 2021, 7, 93. [CrossRef]

46. Sauer, F. Die Temperaturabhängigkeit von Dispersionskräften. Ph.D. Thesis, Universität Göttingen, Göttingen, Germany, 1962.

47. Mehra, J. Temperature correction to the Casimir effect. Physica 1967, 37, 145-152. [CrossRef]

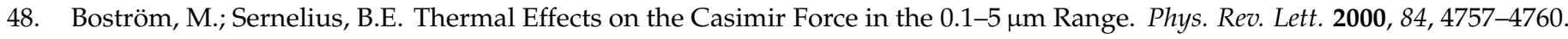
[CrossRef]

49. Genet, C.; Lambrecht, A.; Reynaud, S. Temperature dependence of the Casimir effect between metallic mirrors. Phys. Rev. A 2000, 62, 012110. [CrossRef]

50. Ingold, G.-L.; Lambrecht, A.; Reynaud, S. Quantum dissipative Brownian motion and the Casimir effect. Phys. Rev. E 2009, 80, 041113. [CrossRef] [PubMed]

51. Parsegian, V.A.; Ninham, B.W. Temperature-dependent van der Waals forces. Biophys. J. 1970, 10, 664-674. [CrossRef]

52. Weber, A.; Gies, H. Nonmonotonic Thermal Casimir Force from Geometry-Temperature Interplay. Phys. Rev. Lett. 2010, 105, 040403. [CrossRef] [PubMed]

53. Weber, A.; Gies, H. Geothermal Casimir phenomena for the sphere-plate and cylinder-plate configurations. Phys. Rev. D 2010, 82, 125019. [CrossRef]

54. Canaguier-Durand, A.; Maia Neto, P.A.; Lambrecht, A.; Reynaud, S. Thermal Casimir Effect in the Plane-Sphere Geometry. Phys. Rev. Lett. 2010, 104, 040403. [CrossRef] [PubMed]

55. Canaguier-Durand, A.; Maia Neto, P.A.; Lambrecht, A.; Reynaud, S. Thermal Casimir Effect for Drude metals in the plane-sphere geometry. Phys. Rev. A 2010, 82, 012511. [CrossRef]

56. Zandi, R.; Emig, T.; Mohideen, U. Quantum and thermal Casimir interaction between a sphere and a plate: Comparison of Drude and plasma models. Phys. Rev. B 2010, 82, 195423. [CrossRef]

57. Rodriguez-Lopez, P. Casimir energy and entropy in the sphere-sphere geometry. Phys. Rev. B 2011, 84, 075431. [CrossRef]

58. Umrath, S.; Hartmann, M.; Ingold, G.-L.; Maia Neto, P.A. Disentangling geometric and dissipative origins of negative Casimir entropies. Phys. Rev. E 2015, 92, 042125. [CrossRef] [PubMed]

59. Bimonte, G.; Emig, T. Exact Results for Classical Casimir Interactions: Dirichlet and Drude Model in the Sphere-Sphere and Sphere-Plane Geometry. Phys. Rev. Lett. 2012, 109, 160403. [CrossRef]

60. Bimonte, G. Classical Casimir interaction of a perfectly conducting sphere and plate. Phys. Rev. D 2017, 95, 065004. [CrossRef]

61. Bimonte, G. Beyond-proximity-force-approximation Casimir force between two spheres at finite temperature. II. Plasma versus Drude modeling, grounded versus isolated spheres. Phys. Rev. D 2018, 98, 105004. [CrossRef]

62. Bordag, M.; Pirozhenko, I. Vacuum energy between a sphere and a plane at finite temperature. Phys. Rev. D 2010, 81, 085023 . [CrossRef]

63. Fosco, C.D.; Lombardo, F.C.; Mazzitelli, F.D. Proximity force approximation for the Casimir energy as a derivative expansion. Phys. Rev. D 2011, 84, 105031. [CrossRef]

64. Fosco, C.D.; Lombardo, F.C.; Mazzitelli, F.D. Derivative-expansion approach to the interaction between close surfaces. Phys. Rev. A 2014, 89, 062120. [CrossRef]

65. Fosco, C.D.; Lombardo, F.C.; Mazzitelli, F.D. Derivative expansion for the Casimir effect at zero and finite temperature in $d+1$ dimensions. Phys. Rev. D 2012, 86, 045021. [CrossRef]

66. Fosco, C.D.; Lombardo, F.C.; Mazzitelli, F.D. Derivative expansion for the electromagnetic Casimir free energy at high temperatures. Phys. Rev. D 2015, 92, 125007. [CrossRef]

67. Canaguier-Durand, A.; Ingold, G.-L.; Jaekel, M.T.; Lambrecht, A.; Maia Neto, P.A.; Reynaud, S. Classical Casimir interaction in the plane-sphere geometry. Phys. Rev. A 2012, 85, 052501. [CrossRef]

68. Nieto-Vesperinas, M. Scattering and Diffraction in Physical Optics; World Scientific: Singapore, 2006. [CrossRef]

69. Landau, E. Handbuch der Lehre von der Verteilung der Primzahlen; B. G. Teubner: Leipzig, Germany, 1909 ; p. 60.

70. Schoger, T.; Ingold, G.-L. Classical Casimir free energy for two Drude spheres of arbitrary radii: A plane-wave approach. SciPost Phys. 2021, 4, 011. [CrossRef]

71. Olver, F.W.J.; Daalhuis, A.B.; Lozier, D.W.; Schneider, B.I.; Boisvert, R.F.; Clark, C.W.; Miller, B.R.; Saunders, B.V.; Cohl, H.S.; McClain, M.A. (Eds.) NIST Digital Library of Mathematical Functions. Available online: http://dlmf.nist.gov/ (accessed on 1 May 2021).

72. Paris, R.B. The evaluation of single Bessel function sums. Math. Æterna 2018, 8, 71-82.

73. Bordag, M.; Nikolaev, V. First analytic correction beyond the proximity force approximation in the Casimir effect for the electromagnetic field in sphere-plane geometry. Phys. Rev. D 2010, 81, 065011. [CrossRef]

74. Henning, V.; Spreng, B.; Maia Neto, P.A.; Ingold, G.L. Data for “Casimir interaction between a plane and a sphere: Correction to the proximity-force approximation at intermediate temperatures". Zenodo 2021. [CrossRef]

75. Bohren, C.F.; Huffman, D.R. Absorption and Scattering of Light by Small Particles; Wiley-VCH: Weinheim, Germany, $2004 ;$ Chapter 4. [CrossRef] 
76. Temme, N.M. Asymptotic Methods for Integrals; Series in Analysis; World Scientific: Singapore, 2015; Volume 6. [CrossRef]

77. Spreng, B. Plane-Wave Approach to the Casimir Interaction between Colloid Particles. Ph.D. Thesis, Universität Augsburg, Augsburg, Germany, 2020.

78. Nussenzveig, H.M. High-Frequency Scattering by an Impenetrable Sphere. Ann. Phys. 1965, 34, 23. [CrossRef] 